\title{
OTSU's Thresholding with supervised learning approach for cancer lesion detection
}

\author{
S.Asif Hussain ${ }^{1}$, Dr. D. Satya Narayana ${ }^{2}$, Dr. M.N. Giri Prasad ${ }^{3}$ \\ ${ }^{I}$ Department of ECE, AITS, Rajampet, Andhra Pradesh, India. \\ ${ }^{2}$ Department of ECE, RGMCET, Nandyal, Andhra Pradesh, India. \\ ${ }^{3}$ Department of ECE, JNTUA, Anantapur, Andhra Pradesh, India.
}

\begin{abstract}
For Tracking interfaces and shapes which depends on the regions of pixel intensity is a challenging task in image segmentation. Many level set methods have been formulated for region based and edge based models in computer aided diagnosis systems. In order to provide accurate modeling involving numerical computations, contours, lesions and bias variance which often rely on pixel intensity variations for the region of Interest. The proposed method involves the formulation by deriving a global criterion function in terms of neighborhood pixels to represent domain field and bias variance characteristics. Gaussian impulse is used for smoothening sharp edges. Computational neural networks provide the integral part of most learning algorithms as images consists of redundant attributes of data which have redundant network connections with different input patterns of small weights form a network training process for minimizing the energy and to estimate the bias field correction for various imaging modalities. In order to have valuable diagnostic information in disease diagnosis the extracted features of PET and CT images are modeled with neural networks. The trained data sets are useful in removing artifacts and providing resultant ROI.
\end{abstract}

Keywords; Contours, Gaussian impulse,Redundant attributes, bias variance

\section{INTRODUCTION}

The scope of biomedical imaging covers data acquisition, image reconstruction, and image analysis, involving theories, methods, systems, and applications. While tomography and post-processing techniques become increasingly sophisticated, traditional and emerging modalities play more and more critical roles in anatomical, functional, cellular, and molecular imaging [1]. The overall goal of the International Journal of Biomedical Imaging is to promote research and development of biomedical imaging by publishing high-quality research articles and reviews in this rapidly growing interdisciplinary field.

Images of the human body are generated for clinical use or medical science by a method called medical imaging. Normally the medical imaging is not used to refer to the procedure of imaging removed organs and tissues although they can be imaged for medical reasons. Several imaging techniques are based on reconstructing an image as a set of projections of the imaged object from data either by direct interpretation of data or subsequent to certain preprocessing.

An active research field in modern times is the tomography image reconstruction. Computerized tomography (CT), positron emission tomography (PET), and magnetic resonance imaging (MRI) and several others employ iterative reconstruction as a collection of methods for reconstructing two dimensional and three dimensional images from the projections of the object. Multiple views of the inside of the body is generated by Computed Tomography (CT) scanning using special x-ray equipments [2]. Computer systems are used to reconstruct these multiple $\mathrm{x}$-ray views into cross-sectional images of the body. Filtered back-projection (FBP) is used for permitting image reconstruction of gated myocardial perfusion studies in all general SPECT camera software packages.

Medical imaging has been undergoing a revolution in the past decade with the advent of faster, more accurate, and less invasive devices. This has driven the need for corresponding software development which in turn has provided a major impetus for new algorithms in signal and image processing. Many of these algorithms are based on partial differential equations and curvature driven flows which will be the main topics of this survey paper.

Mathematical models are the foundation of biomedical computing. Basing those models on data extracted from images continues to be a fundamental technique for achieving scientific progress in experimental, clinical, biomedical, and behavioral research. Today, medical images are acquired by a range of techniques across all biological scales, which go far beyond the visible light photographs and microscope images of the early 20th century.

Modern medical images may be considered to be geometrically arranged arrays of data samples which quantify such diverse physical phenomena as the time variation of hemoglobin de oxygenation during neuronal 
metabolism, or the diffusion of water molecules through and within tissue [3]. The broadening scope of imaging as a way to organize our observations of the biophysical world has led to a dramatic increase in our ability to apply new processing techniques and to combine multiple channels of data into sophisticated and complex mathematical models of physiological function and dysfunction.

\section{A. Existing System}

\section{BACKGROUND}

In this Existing methodology the method proposed by Zhang et al. For estimating the field distortion and simultaneously segmenting an MRI image and provide implementation details on how it has been adapted to work with MRI images. This method essentially estimates the low (spatial)-frequency multiplicative degradation field while at the same time identifying regions of similar intensity inhomogeneity using an MRFMAP frame work [4]. Although it is developed for another imaging modality, under simplified assumptions, this can be justified using the same approach on displayed CT and PET in Proposed methodology.

\section{B. criterion for filtering edge information}

It is assumed that interpolated boundaries will partially overlap with the true edges found using edge detection. The probability of edges overlapping with shape-interpolated boundaries may be modelled using Bayes' probability. It is assumed that the probability of overlap at interpolated slices is greater than or equal to that at user-initialized contours.

Edges are divided into edge components based on their connectivity. To retain edges with higher saliency, the edge components are sorted in descending order relative to the amount of overlap with the boundary. When the cumulative probability of overlap exceeds that obtained from user initialized contours, the remaining edge components are discarded [5]. The Bayes' classification is thus not employed for training, but rather as a guide to how well boundaries can be defined based on edge detection.

To achieve edge correspondence for interpolated boundaries Bayesian Criterion function is discussed in proposed methodology.

\section{c. Local Intensity Clustering Property:}

Region-based image segmentation methods typically rely on a specific region descriptor (e.g. intensity mean or a Gaussian distribution) of the intensities in each region to be segmented. However, it is difficult to give such a region descriptor for images with intensity in homogeneities [6]. Moreover, intensity in homogeneities often lead to overlap between the distributions of the intensities in the regions Therefore, it is impossible to segment these regions directly based on the pixel intensities .Nevertheless, the property of local intensities is simple, which can be effectively exploited in the formulation of our method for image segmentation with simultaneous estimation of the bias field based on the image model and the assumptions A1 and A2, we are able to derive a useful property of local intensities, which is referred to as a local intensity clustering property as described and justified below. To be specific, we consider a circular neighborhood with a radius centered at each point defined The partition of the entire domain induces a partition of the neighbourhood i.e., forms a partition of For as lowly varying bias field the values for all in the circular Neighborhood are close to i.e. for

$$
b(X) \approx b(y) \quad \text { for } X \in \phi_{j}
$$

Thus, the intensities in each sub region are close to the constant i.e.

$$
b(X) J(X) \approx b(y) c_{i} \quad \text { for } \quad X \in \phi_{y}
$$

\section{PROPOSED ALGORITHM}

To make work with CT images Simplified assumptions are made in proposed methodology to achieve criterion function for the boundaries model specification, Boundary-edge correspondence Local Intensity Clustering Property, Energy formulation and Multiphase Level Set Formulation are to be assumed.

\section{A. Model Specification:}

Let $\mathrm{S}$ be a lattice indexing the pixels in the given image. Further,

$$
I=\left(I_{1} \ldots \ldots, I_{N}\right) \text { and } I^{*}=\left(I_{1}^{*} \ldots \ldots . I_{N}^{*}\right) \ldots \ldots \ldots(1)
$$

Let and be the observed and the ideal (that is, without intensity in homogeneity distortion) intensities of the given image respectively, being the number of pixels in the image. We assume that the distortion at pixel can be expressed by a multiplicative model of the form [7]

$$
I_{i}=I_{i}^{*} \times d_{i}
$$


Where represents the gain of the intensity due to the intensity in homogeneity at pixel A logarithmic transformation of this equation yields an addition the observed and the ideal log-transformed intensities, then

$$
\mathrm{y}=\mathrm{y}^{*}+\mathrm{d}
$$

Where denotes the log-transformed intensity distortion field. Segmentation can be considered as a problem of statistical classification, which is to assign every pixel a class label from a label set. Let denote the label set.

A labeling of will be denoted by in which is the corresponding class label of pixel Given the class

label, it is assumed that the intensity value at pixel follows a Gaussian distribution(this assumption will be justified in Section III) with parameter being the mean and the variance of class respectively[8]

$$
p\left(y_{i}^{*} \| x_{i}\right)=g\left(y_{i}^{*} ; \theta\left(x_{i}\right)\right)
$$

Bayes' rule can be used to obtain the posterior probability of the distortion field, given the observed intensity values

$$
P(d \| y)=p(y \mid d) p(d) / P(y)
$$

Where is normalization constant The prior probability density of the distortion field is modeled as a Gaussian with zero mean to capture its smoothness property. The maximum a posterior (MAP) principle can be employed to obtain the optimal estimate of the distortion field, given the observed intensity values[9]

Solving this equation leads to the update equations (see for detail)

$$
w_{i j}=\frac{p\left(y_{i} \| x_{i}, d_{i}\right) p\left(x_{i}=j\right)}{p\left(y_{i} \| d_{i}\right)} \ldots \ldots .
$$

Here, is the posterior probability that pixel belongs to class given the distortion field estimate, is a low-pass filter, is the mean residual in which for pixel And $\psi$ is the mean inverse covariance, in which if otherwise.

$$
\psi_{i k}^{-1}=\left\{\sum_{0}^{j=L} w_{i j} \sigma_{j}^{-2} \quad f_{i}=K\right.
$$

\section{B. Otsu's Thresholding Method:}

Otsu's method is used to automatically perform clustering-based image thresholding or the reduction of a gray level image to a binary image. The algorithm assumes that the image to be threshold contains two classes of pixels or bi-modal histogram (e.g. foreground and background) then calculates the optimum threshold separating those two classes so that their combined spread (intra-class variance) is minimal.

The threshold is that which minimizes the weighted within-class variance which in turns out to be the same as maximizing the between-class variance Operates directly on the gray level histogram. Some of the Otsu's assumptions which can be defined are Histogram (and the image) is bimodal. No use of spatial coherence, nor any other notion of object structure. This Assumes stationary statistics, but can be modified to be locally adaptive. One more Assumption is uniform illumination so the bimodal brightness behavior arises from object appearance differences only. The weighted within-class variance is[10]

$$
\sigma_{w}^{2}(t)=q_{1}(t) \sigma_{1}^{2}(t)+q_{2}(t) \sigma_{2}^{2}(t)
$$

Where the class probabilities are estimated as

$$
\mathrm{q}_{1}(\mathrm{t})=\sum_{\mathrm{i}=1}^{\mathrm{t}} \mathrm{p}(\mathrm{i}) \quad \mathrm{q}_{2}(\mathrm{t})=\sum_{\mathrm{i}=\mathrm{t}+1}^{\mathrm{I}} \mathrm{p}(\mathrm{i})
$$

And the class means are given by

$$
\mu_{1}=\sum_{i=1}^{t} \frac{i p(i)}{q_{1}(t)} \mu_{2}=\sum_{i=1}^{t} \frac{i p(i)}{q_{2}(t)}
$$

All it is need to do is just run through the full range of values $[1,256]$ and pick the value that minimizes. But the relationship between the within-class and between-class variances can be exploited to generate recursion relation that permits a much faster calculation.

The basic idea is that the total variance does not depend on threshold (obviously). For any given threshold, the total variance is the sum of the within-class variances (weighted) and the between class variance, which is the sum of weighted squared distances between the class means and the grand mean. After some algebra, we can express the total variance as since the total is constant and independent of $t$, the effect of changing the threshold is merely to move the contributions of the two terms back and forth. So, minimizing the 
within-class variance is the same as maximizing the between-class variance. The nice thing about this is that we can compute the quantities in recursively as we run through the range of $t$ values.

\section{Analytic and Iterative methods:}

Image Reconstruction is broadly classified in two groups which are as follows: Analytical reconstruction and iterative reconstruction are two major types of methods that exist in image reconstruction. Due to computational efficiency and numerical stability, the filtered back projection (FBP) based methods are the type of analytical reconstruction that are extensively utilized at present on clinical CT scanners.

Inverse matrix, Filtered back projection (FBP) and Fourier transform (FT).are the three types of analytical algorithms and traditional algebraic methods and statistical reconstruction methods are the two types of iterative algorithms [11]. Traditional algebraic methods are further categorized into Algebraic reconstruction technique (ART), Simultaneous iterative reconstruction technique (SIRT) and Iterative least-squares technique (ILST). Statistical reconstruction methods are also further categorized into Gradient and Conjugate gradient (CG), Maximum likelihood expectation maximization (MLEM), Ordered-subsets expectation maximization (OSEM), Maximum a posteriori (MAP). Iterative reconstruction has several merits over traditional FBP techniques and hence CT has attracted considerable attention in recent times.

An attractive field normally in digital image processing techniques and particularly in biomedical imaging is 3D image reconstruction The development of fast algorithms, like ordered-subset, expectationmaximization algorithm have partially solved the high computational cost of iterative 3D image reconstruction which is its major demerit. An equivalent but mathematically simpler method to the practically few systems using Radon Transform is back projection. The back-projection algorithm is an efficient algorithm with relative low computer cost developed by Barber and Brown in 1983. The memory accessing times can be decreased many times to once by a parallel back projection algorithm. The reasonably effective deterministic filtered back projection (FBP) is the most common method for complete projection data with high SNR[12]. A Numerous scientific, medical, and technical fields have studied the problem of image reconstruction from projections for several years. Deterministic or statistical methods can be attempted for image reconstruction from projections. Traditionally, filtered back-projection (FBP) algorithms are commonly used to reconstruct PET images. Further, slow time and spatially-dependent filtering step are the major reasons for the computational complexity of these GFBP methods.

Data acquired by moving radiation detector systems are used to form images computationally by dynamic reconstruction. Image is related to the data by system model. A physical, biological or information system is mathematically signified by a model. Models permit to reasoning related to a system and make forecasts about the behavior of a system. Quantitative errors are decreased and spatial resolution of the reconstructions is improved when precise system modeling and anatomical information are used in a clinically feasible reconstruction algorithm. The Parameterized models may have the potential to generate superior results as they encode stronger prior information.

\section{Energy Formulation:}

The above described is local intensity clustering property indicates that the intensities in the neighborhood can be classified in to clusters with centers $m_{i}=b(y)$. This allows us to apply the standard $\mathrm{K}$ means clustering to classify these local intensities. Specifically, for the intensities in the neighborhood the Kmeans algorithm is an iterative process to minimize the clustering criterion [19] continuous form as [13]

$$
f_{y}=\sum_{i=1}^{N} \int_{\Phi y} I(X)-m_{i}^{2} u_{i}(X) d x
$$

Which is the cluster center of the cluster, is the and for since is the membership function of the region, we can rewrite as[14]

$$
F_{y}=\sum_{i=1}^{N} \int_{\Omega \phi y \cap} I d x \ldots \ldots
$$

In view of the clustering criterion in and the approximation of the cluster centre which is defined as clustering criterion for classifying the intensities which is introduced as a nonnegative window function, also called kernel function, such that for With the window function, the clustering criterion function can be rewritten as[15]

$$
\varepsilon_{y}=\sum_{i=1}^{N} \int_{\Omega i} K(y-x) I(X)-b(y) c_{i}^{2} d x
$$

This local clustering criterion function is a basic element in the formulation of our method. 


\section{E. Boundary-edge correspondence:}

Ideally, the match between boundary and edge should be one-to-one. However, deviations in the interpolated shape will not initialize $B i$ well. To prevent many-to-one snapping of boundary points, a minimum snapping-distance map is stored for every edge point. Subsequent boundary points will only be allowed to snap to the edge point if the snapping distance is less than or equal to the value in the minimum snapping-distance map. Therefore, boundary points will not arbitrarily snap to false edges if there are no edges to be found. During the first iteration, the search window has not been adaptively altered to match the edge proximity for the image slice. There is a possibility that a false edge will be included in the Bayesian criterion. To prevent this, an inverse weighted distance transform, $M$, is multiplied to $F i_{, k}$, where $M$ is a square matrix. Denoting $M_{p q}$ as an element in $M$ and any two points on the $B i$ as $b_{p}$ and $b_{q \cdot}, p q M$ is defined in Eq. 1[16].

$$
M_{p q}=\frac{1}{\left|b_{p}-b_{q}\right|+1} \ldots \ldots . .(14) .
$$

\section{F. Pre-processing:}

This methods use a small neighbor hood of a pixel in an input image to get a new brightness value in the output image such preprocessing operations is also called filtration. Local pre-processing methods can be divided into two groups according to the goal of the processing. Smoothing suppresses noise or other small fluctuations in the image; equivalent to the suppression of high frequencies in the frequency domain. Unfortunately, smoothing also blurs all sharp edges that bear important information about the image. Gradient operators are based on local derivatives of the image function. Derivatives are bigger at locations of the image where the image function undergoes rapid changes[17]. The aim of gradient operators is to indicate such locations in the image. Gradient operators suppress low frequencies in the frequency domain (i.e. they act as high-pass filters). Noise is often high frequency in nature; unfortunately, if a gradient operator is applied to an image the noise level increases simultaneously. Gradient operators permit smoothing and edge enhancement simultaneously.

\section{G. Proposed Block diagram:}

Each block is defined in different steps to describe Visual quality of CT images

1) Input image: The Input image is carried with the CT image or PET image to provide motion of the patient. For identifying the Region of Interest in CT motion images. The given input image is automatically subjected in generation of the sinograms to get rough data and estimation about the image. In the simplest form of CT imaging only the desired cross sectional plane of the body is irradiated using a finely collimated ray of X-ray photons. Ray integrals are measured at many positions and angles around the body scanning the body in the process. The principle of image reconstruction from projections is then used to compute an image of a section of the body hence the name computed tomography

2) Preprocessing: In the above block diagram this step is carried out with CT images to avoid noise corruptions and make the data more accurate and provide better illusion with appropriate amount of Intensity in the next step rough data is generated.[18]

3) Image Smoothening: Gaussian noise impulse is used to smoothen the region of interest in the center part of the image.

4) Image Enhancement: The principle objective of enhancement techniques is to process an image, so that the result is more suitable than the original image for a specific application. The approaches are divided into two types is Spatial domain method and Frequency domain method. The spatial domain refers to the image plane itself and approaches in this category are based on direct manipulation of pixels in an image. The frequency domain processing techniques are based on modifying the Fourier transformation of an image. For the given results shown frequency domain processing techniques are used.

5) Blurring Initial Check Point: To identify the artifacts and to detect the anomalies in the image blurring from initial point with spatial mask is used.

6) Bias Correction: To smoothen the image in center part to signify the difference from edge boundaries and the resulted is extremely accurate solution with more efficient optimization techniques

7) Iteration count: To identify the of Interest (ROI) from initial point 50 iterations are performed.

8) Region of Interest: This region gives the detected anomalies for specific measures.

9)Quantitative Measures: Some of the qualitative measures have been performed on the obtained images, to check for the overall signal to noise ratio to identify visual artifacts to have computational measures of the process to define image quality metrics

10) Classification Methods

Selected Features are applied on the classifier the recognition ability of the classifiers depends on the choice of the used diagnostic features as well as the available training data.

- Feed forward Back Propagation Neural Network 
- Support Vector Machine

11) Feed Forward Back Propagation Neural Network:

Neural Network is a set of connected input/output units where each connection has weight associated with it the number of units in the hidden layer and the number of hidden layers depends on implementation. The output layer has as many units as the number of classes. The network is feed forward in that none of the weights cycles back to an input unit or to an output unit of a previous layer. Back propagation learns by iteratively processing a set of training data [19]. It is a case of supervised Classification learning, neural network learns by adjusting the weights so as to be able to correctly classify the training data and hence after testing phase to classify unknown data.

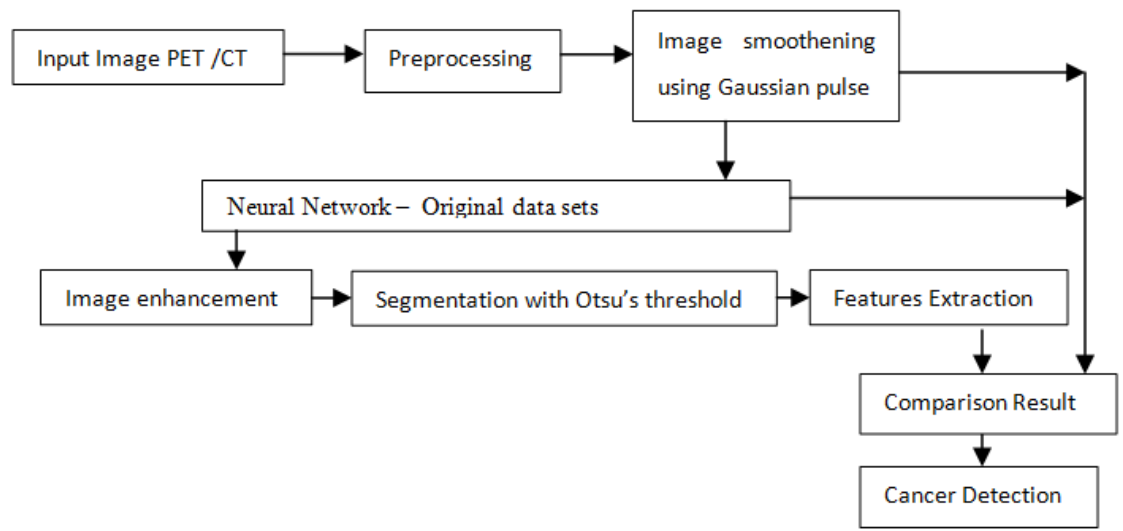

Fig.1. The above block diagram describes cancer lesion detection from the given CT/PET image

IV.

EXPERIMENT SimUlation AND RESULT ANALYSIS

To verify the performance of the proposed segmentation algorithm, we experiment with color remote sensing images and natural images. We show results of some of these experiments. Figure. 9 and Figure.10 show some typical results of our color image segmentation algorithm.

In fact, we can compare our algorithm and traditional SRG algorithm here. Our method use region rather than pixel as initial seeds. In this sense, high-level knowledge of the image partitions can be exploited through the choice of the seeds much better because region has more information compared to pixels.

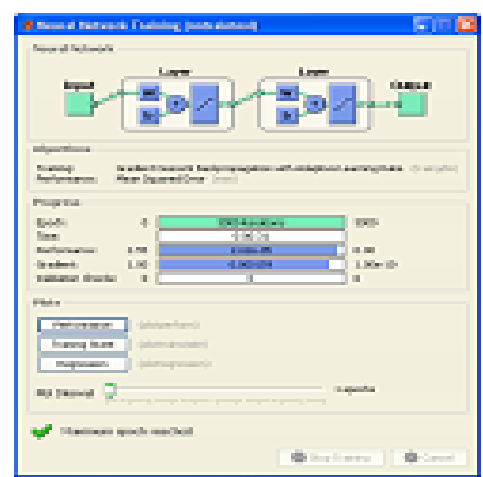

(a)

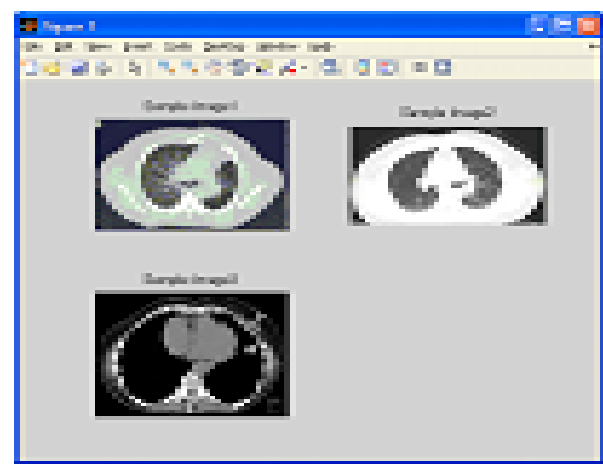

(b)

Fig.2. (a) Neural Network model using Feed forward (b) GUI Model showing input images of CT \& PET

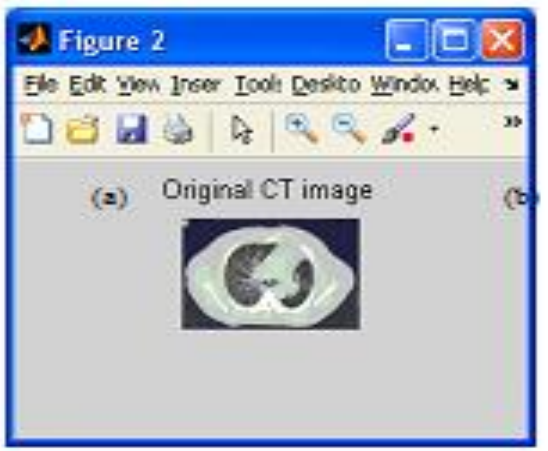

(a)

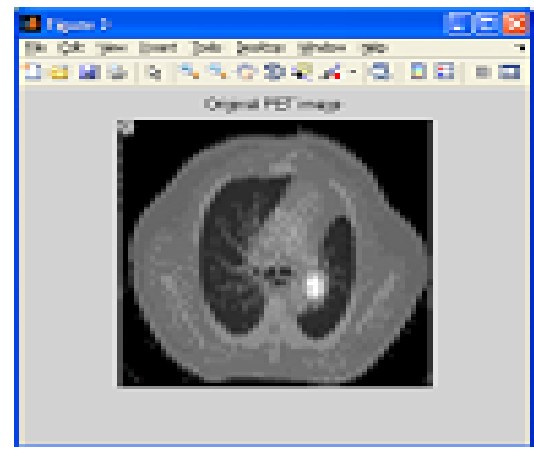

(b)

Fig.3. (a) Input CT Image (b) PET image as Input 


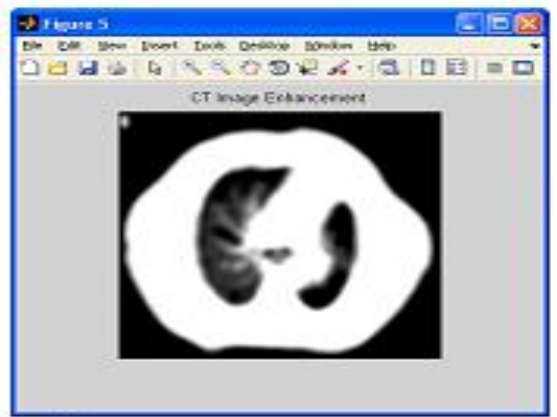

(a)

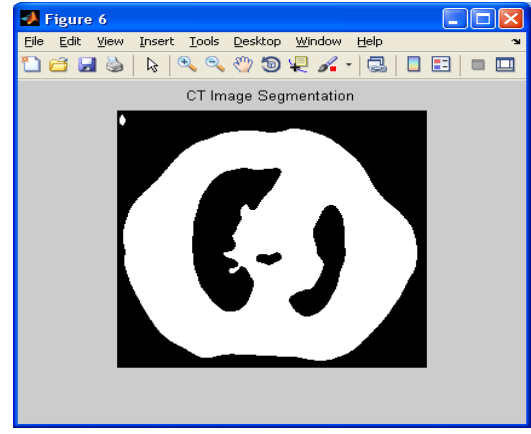

(b)

Fig.4. (a) CT Image Enhancement (b) CT Image Segmentation

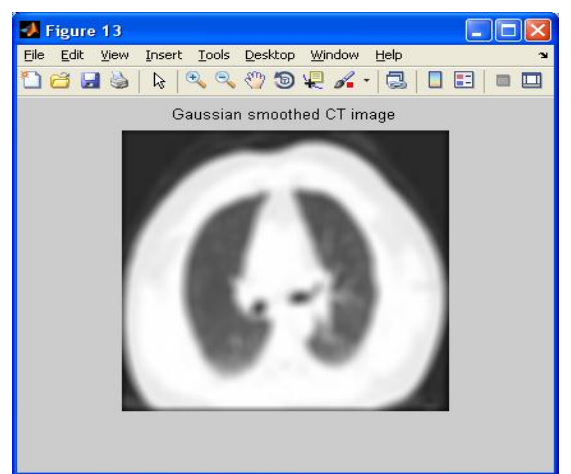

(a)

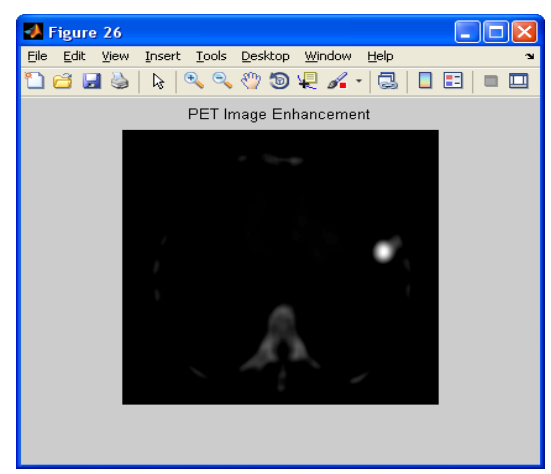

(b)

Fig.5. (a) Gaussian Smoothened CT Image (b) PET Image Enhancement

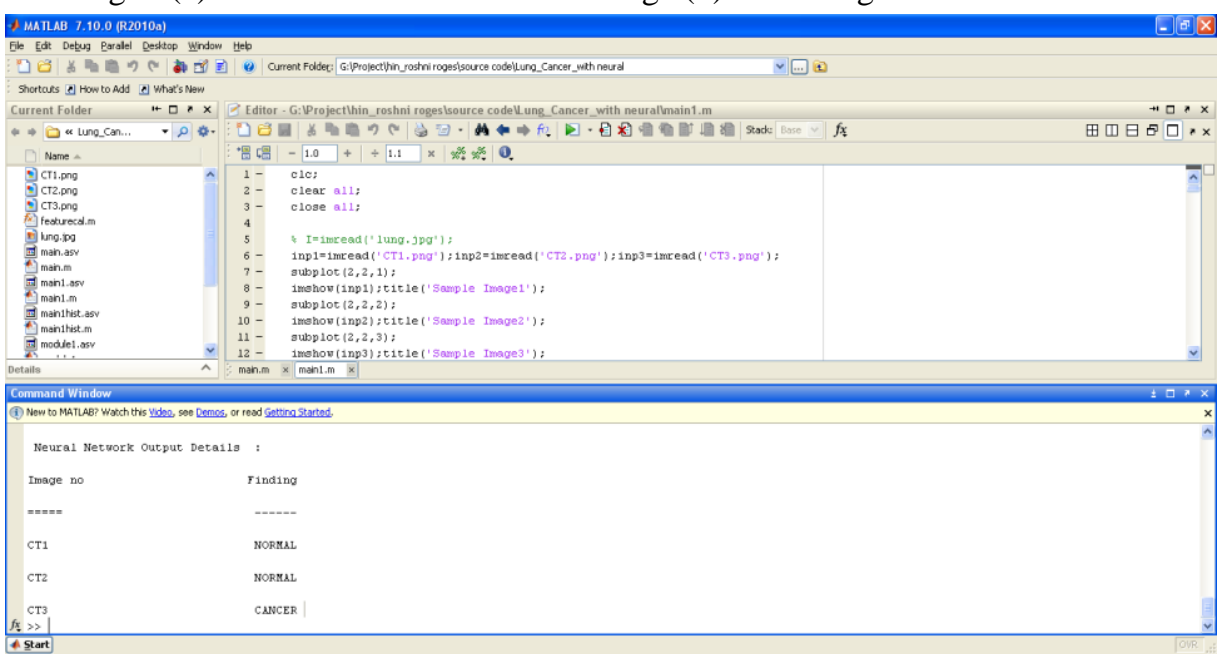

Fig.6. Describes the neural network output details when performed training

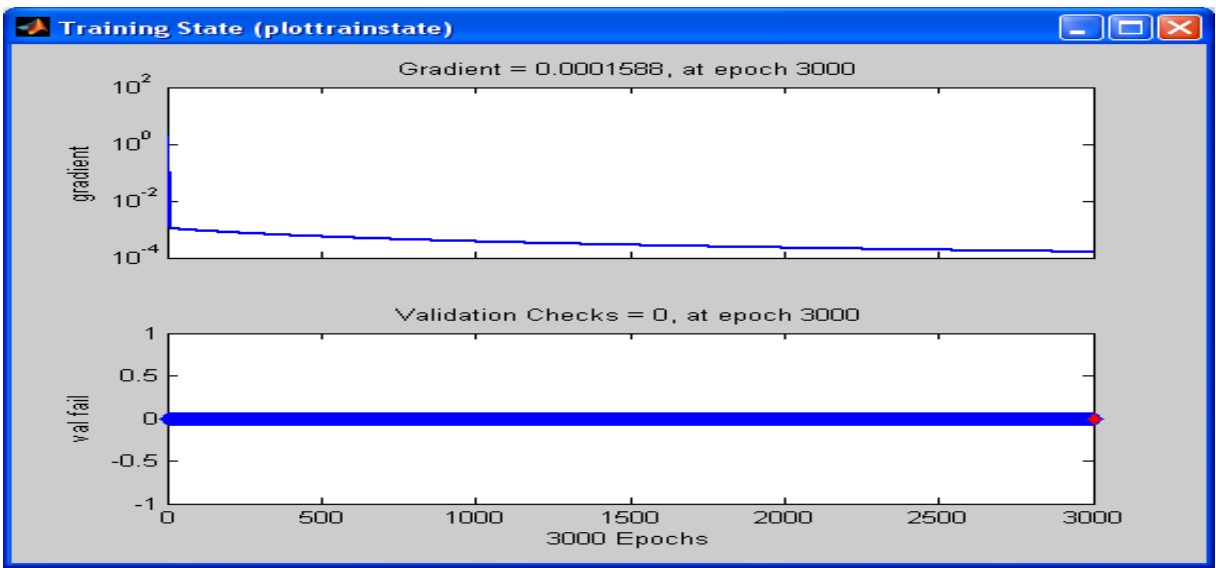

Fig.7. Performance measures for the training state 


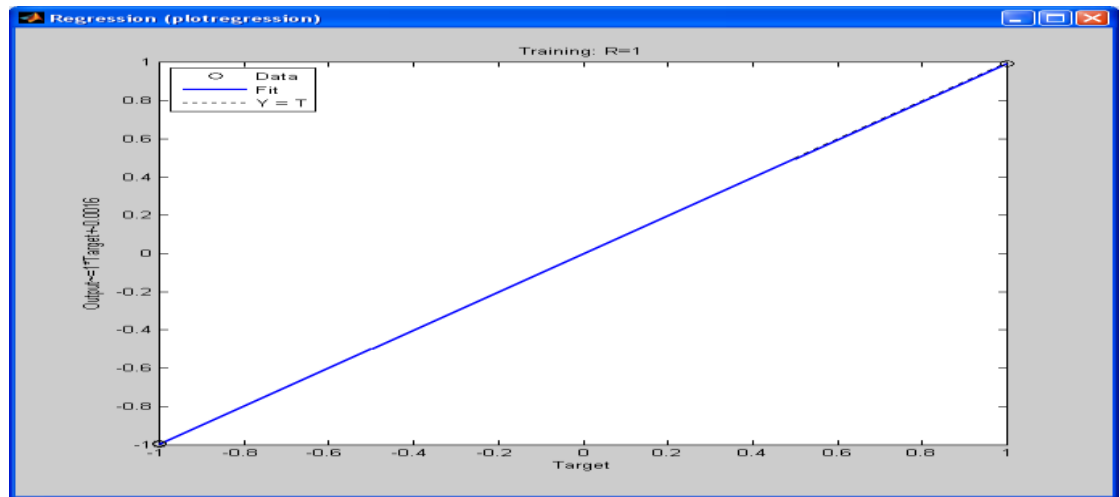

Fig.8. shows a regression state for given training state

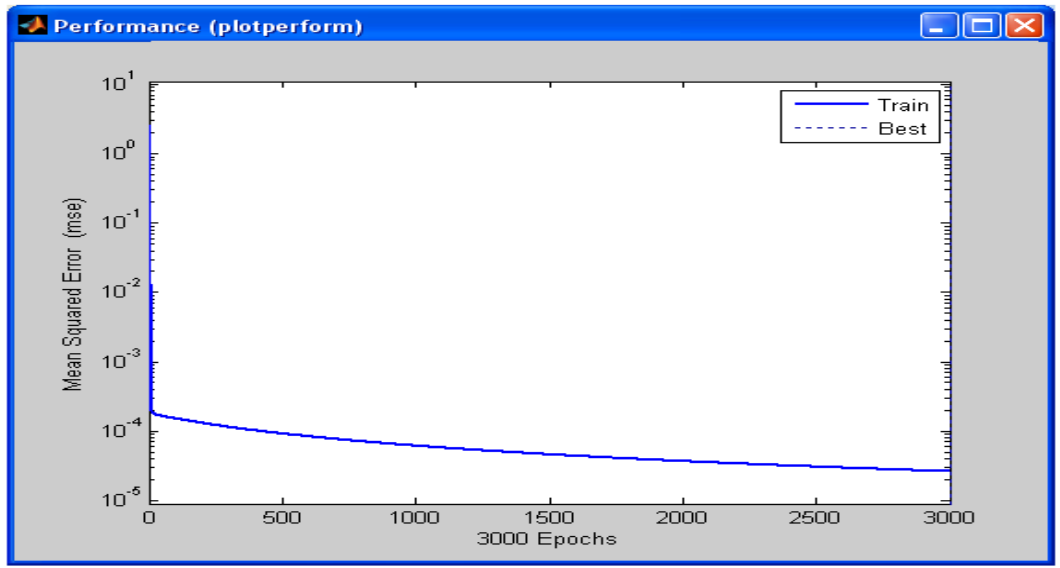

Fig.9. shows the performance of neural network output

\section{CONCLUSION}

This work presents a variation level set framework for segmentation and bias correction of images with intensity in homogeneities. Based on a generally accepted model of images with intensity in homogeneities and a derived local intensity clustering property, the work defines energy of the level set functions that represent a partition of the image domain and a bias field that accounts for the intensity in homogeneity. Segmentation and bias field estimation are therefore jointly performed by minimizing the proposed energy functional. The slowly varying property of the bias field derived from the proposed energy is naturally ensured by the data term in our variation framework, without the need to impose an explicit smoothing term on the bias field. The proposed method is much more robust to initialization than the piecewise smooth model. Experimental results have demonstrated superior performance of our method in terms of accuracy, efficiency, and robustness.

\section{Acknowledgements}

This paper is fully supported by the Research Promotion Scheme of AICTE New Delhi, India (F.No:8023/RID/RPS-2/2010-11).

\section{REFERENCES}

[1] S. Asif Hussain, Dr. M. N. Giri Prasad and Dr. D. Satya Narayana "A novel Feature Selection mechanism for medical image retrieval system” International Journal of Advances in Engineering \& Technology(IJAET), Volume 6 Issue 3, pp. 1283-1298, July 2013, ISSN: 2231-1963 DOI:10.7323.

[2] S. Asif Hussain, Dr. M. N. Giri Prasad and Dr. D. Satya Narayana "Superior Reconstruction Quality improvement of CT Image for Bias Correction Variance Measures"- International Journal of Computer Applications 2012- IJCA July 2012 Published by Foundation of Computer Science, New York, USA., Proceedings in Volume 47 - Number 5 of IJCA July 2012 ISBN: $973-93-$ 80868-79-4 ISSN: 0975 - 888 DOI 10.5120/7185-9918.

[3] S. Asif Hussain, Dr. M. N. Giri Prasad and Dr. D. Satyanarayana "3D Medical Image Modeling with Dynamic Image Reconstruction"- International Journal of Engineering Research and Technology. 2012- IJERT July 2012 Published by International Research Publication House, Proceedings in Volume 5, Number 3 (APRIL 2012), pp. 213-217 ISSN 0974-3154.

[4] Olabarriaga, S.D. and Smeulders, A.W.M., "Interaction in the Segmentation of Medical Images: A Survey", Med. Image Analysis, 5: 127-142, 2001.

[5] Osher, S. and Sethian, J.A., "Fronts Propagating with Curvature Dependent Speed: Algorithms Based on Hamilton-Jacobi Formulations", J. Comp. Physics, 79:12- 49, 1988.

[6] P. N. T.Wells and M. Halliwell, "Speckle in ultrasonic imaging," Ultrasonics, vol. 19, pp. 225-229, 1981. 
[7] A. N. Evans and M. S. Nixon, "Biased motion-adaptive temporal filteringfor speckle-reduction in echocardiography," IEEE Trans. Med.Imag., vol. 15, pp. 39-50, Feb. 1996.

[8] V. Caselles, R. Kimmel, and G. Sapiro, “Geodesic active contours,"Int. J. Comput. Vis., vol. 22, no. 1, pp. 61-79, Feb. 1997.

[9] T. Chan and L. Vese, “Active contours without edges,” IEEE Trans.Image.Process., vol. 10, no. 2, pp. 266-277, Feb.2001.

[10] S. Kichenassamy, A. Kumar, P. Olver, A. Tannenbaum, and A. Yezzi, "Gradient flows and geometric active contour models," in Proc. 5th Int.Conf. Comput. Vis., 1995, pp. 810-815.

[11] R. Kimmel, A. Amir, and A. Bruckstein, "Finding shortest paths onsurfaces using level set propagation," IEEE Trans. Pattern Anal.Mach.Intell., vol. 17, no. 6, pp. 635-640, Jun. 1995.

[12] C. Li, C. Kao, J. C. Gore, and Z. Ding, "Minimization of region-scalablefitting energy for image segmentation," IEEE Trans. ImageProcess., vol. 17, no. 10, pp. 1940-1949, Oct. 2008.

[13] R. Malladi, J. A. Sethian, and B. C.Vemuri, "Shape modeling with frontpropagation: A level set approach," IEEE Trans. Pattern Anal. Mach.Intell., vol. 17, no. 2, pp. 158-175, Feb. 1995.

[14] R. Ronfard, "Region-based strategies for active contour models," Int.J.Comput. Vis., vol. 13, no. 2, pp. 229-251, Oct. 1994.

[15] C. Samson, L. Blanc-Feraud, G. Aubert, and J. Zerubia, "A variationalmodel for image classification and restoration," IEEE Trans. PatternAnal. Mach. Intell., vol. 22, no. 5, pp. 460-472, May 2000.

[16] S. Theodoridis and K.Koutroumbas, Pattern Recognition. NewYork:Academic, 2003.

[17] A. Tsai, A. Yezzi, and A. S.Willsky, "Curve evolution implementationof the Mumford-Shah functional for image segmentation, denoising,interpolation, and magnification,” IEEE Trans. Image Process., vol. 10,no. 8, pp. 1169-1186, Aug. 2001.

[18] A. Vasilevskiy and K. Siddiqi, "Flux-maximizing geometric flows,"IEEE Trans. Pattern Anal. Mach. Intell., vol. 24, no. 12, pp. 1565-1578,Dec. 2002.

[19] L. Vese and T. Chan, "A multiphase level set framework for image segmentation using the Mumford and Shah model," Int. J. Comput. Vis.,vol. 50, no. 3, pp. 271-293, Dec. 2002.

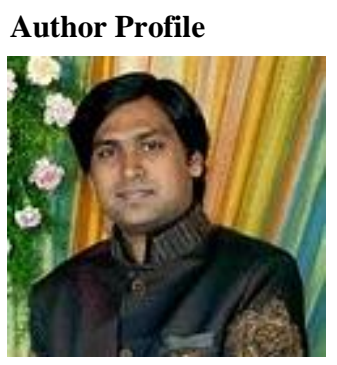

Asif Hussain. Shaik received B.Tech \& M.Tech Degree in Electronics \& Communication Engg. From JNTUniversity, Hyderabad, India. He is currently working towards $\mathrm{PhD}$ Degree in Biomedical Image Processing at JNTU University, Anantapur, India. Presently he is with Annamacharya Institute of Technology \& Sciences, Rajampet, A.P., and India. He is working as Assistant Professor in Dept. of ECE. He presented many research papers in National \& International Conferences\& journals. He is a member of Professional societies like ISTE (India), BMESI (India), IACSIT (Singapore), IAENG (Hongkong) and WASE (Hongkong).His research interests include Signal Processing, Time Series Analysis and Image Processing.

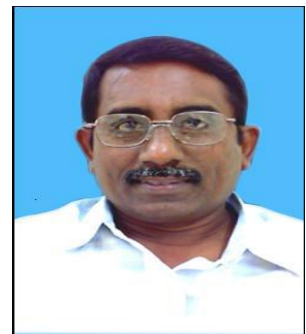

Dr. M. N. Giri Prasad received B.Tech Degree in Electronics \& Communication Engineering from JNT University, Hyderabad, India. He received his M.Tech Degree from S.V.University, Tirupathi, India. He has received $\mathrm{PhD}$ in Biomedical Signal Processing from JNT University, Hyderabad, India. At Present he is Professor in Dept. of ECE at JNTUCE, Anantapur, A.P., India. He also worked as Principal at JNTUCE, Pulivendula, A.P., India. He has more than 20 years of teaching experience. He has more than 30 publications in standard International/technical Journals. His research interests include Biomedical Signal Processing, Antennas and Image Processing. He is a member of Professional societies like IE (India), NAFEN (India), ISTE (India), IACSIT (Singapore),CSTA(USA) and IAENG (Hongkong). He is reviewer of Engineers Australia Technical Journals, Australia, of International Journal of Computer Science and Information Security (IJCSIS), Pittsburgh, USA, International Journal of Electrical, Electronics and Computer Systems (IJEECS), USA and International Journal of Algorithm, of AWEM Group, USA. He is reviewer of National/International Conferences in India and IEEE Sponsored International Conferences held in Singapore and China. He chaired many National/International conferences in India. He is BOS member of reputed engineering colleges in Andhra Pradesh.

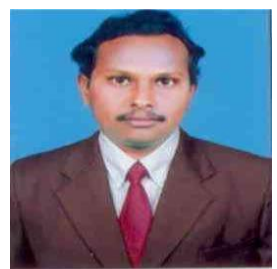

Dr. D. Satyanarayana, Professor in Electronics \& Communication Engineering, having 18 years teaching experience, has been graduate $d$ from Bharatiar University, Coimbatore in the faculty of Electronics \& Communication Engineering in 1992. Later he has obtained his Master's Degree in Digital Systems and Computer Electronics from Jawaharlal Nehru Technology University, Hyderabad in the year 1998. He was awarded a Doctorate for his work in Signal Processing by J.N.T. University, Hyderabad in the year 2009. At present he is heading the department of ECE at RGMEC. He is very actively involved in Research work and presented 10 Research papers on different topics in the field of Electronics. He is a member of Professional societies like ISTE (India), IETE (India) and IEEE. In his total Endeavour as a teacher, he is known for his friendliness with the students and his colleagues. He is a man of simplicity, sincerity and hard working. 